\title{
Development of a Nomogram for Predicting the Occurrence of Symptomatic Intracranial Haemorrhage after Thrombectomy: A Single-Center Retrospective Observational Study
}

\section{YunHui Wang}

Department of Neurosurgery, The People's hospital of Deqing, Huzhou, Zhejiang Province, P.R. China

Qi Tu

the First Affiliated Hospital of Wenzhou Medical University

TingTing Zhao

Department of Neurosurgery, The People's hospital of Deqing, Huzhou, Zhejiang Province, P.R. China

\section{Qiang Ye}

Department of Neurosurgery, The People's hospital of Deqing, Huzhou, Zhejiang Province, P.R. China

\section{Kuang Zheng}

the First Affiliated Hospital of Wenzhou Medical University

\section{WeiZhong Zhang}

the First Affiliated Hospital of Wenzhou Medical University

ZeQun Li ( $\nabla$ lizequn351@sina.com )

the First Affiliated Hospital of Wenzhou Medical University

\section{Research Article}

Keywords: Thrombectomy, Symptomatic Intracranial Hemorrhage, Nomogram, Stroke

Posted Date: February 1st, 2022

DOI: https://doi.org/10.21203/rs.3.rs-1177181/v2

License: (a) (i) This work is licensed under a Creative Commons Attribution 4.0 International License. Read Full License 


\section{Abstract \\ Background}

Symptomatic intracranial haemorrhage $(\mathrm{SICH})$ is a severe and deadly complication in patients with large vessel occlusion (LVO) who receive endovascular treatment (EVT). Recent studies have indicated that many risk factors, including pretreatment scores and the operation process, may be associated with the occurrence of SICH after thrombectomy. This study aims to identify independent risk factors and establish a novel nomogram-based model for patients with anterior LVO to predict the occurrence of SICH after direct thrombectomy or bridge therapy (thrombectomy based on intravenous thrombolysis).

\section{Methods}

Patients with acute ischaemic stroke after EVT to recanalize the blocked artery in anterior circulation were consecutively recruited from November 2017 to March 2019. Baseline information was collected from each patient. These data were subsequently analysed by R Project for Statistical Computing.

\section{Results}

A total of 127 patients with complete data were classified into the training set, among whom 37 patients (29.1\%) fulfilled the criteria for SICH. The results of the multivariate analyses showed that NIHSS $(P=0.024)$, ASPECT $(P<0.001)$ and ASITN $(P=0.017)$ scores were independently associated with the occurrence of SICH after thrombectomy. Ultimately, three independent pretreatment predictors were included in the NIHSS/ASPECT/ASITN (NAA) prediction model, and the receiver operating characteristic analysis results showed an area under the curve (AUC) of 0.845 ( $95 \% \mathrm{Cl}=0.763-0.928)$. The calibration plots showed that the actual observations were consistent with the measured and predicted results of the nomogram.

\section{Conclusions}

In this study, a novel model based on NAA for predicting the occurrence of SICH after thrombectomy in patients with anterior LVO was established and validated internally. The results suggest that this model can help improve perioperative evaluations and individualized treatment strategies.

\section{Introduction}

Intracerebral haemorrhage (ICH) is the major complication after endovascular treatment (EVT) and is classified as symptomatic or asymptomatic according to the new Heidelberg Bleeding Classification. [1] Symptomatic intracerebral haemorrhage (SICH) can occur during thrombectomy or postoperatively within 72 hours and is independently associated with a poor outcome as well as increased morbidity and 
mortality. In a multicentre retrospective analysis of 1122 patients, $\mathrm{SICH}$ was associated with higher mortality (3.53 (2.19 to 5.68), P<0.0001). [2] When a modified Rankin Scale (mRS) score of 0 to 2 was used to define a favourable neurological outcome, fewer patients with $\mathrm{SICH}$ reached a favourable outcome 90 days after the index stroke than patients without $\mathrm{SICH}(8.9 \%$ versus $51.2 \% ; P<0.001)$. [3]

Before the new Heidelberg Bleeding Classification was published, there were different definitions of SICH, including the European Co-operative Acute Stroke Study-II (ECASS-II), National Institute of Neurological Disorders and Stroke (NINDS) and Safe Implementation of Treatment in Stroke-International Stroke Thrombolysis Registry (SITS-ISTR). According to these different definitions, the reported rate of SICH in randomized controlled trials (RCTs) ranged from 3.6-9.3\%. [4-8] Among RCTs, the highest rate of 9.3\% was reported in the THERAPY trial, whereas the SWIFT PRIME, EXTEND-IA and PISTE trials did not report any incidences of $\mathrm{SICH}$. Among non-RCTs, the rates of SICH ranged from 1.9-15.8\%. [9-11] According to the new Heidelberg Bleeding Classification, the rates of $\mathrm{SICH}$ ranged from $16.0-18.6 \%$. $[3,12]$

Recently, baseline clinical characteristics such as high National Institutes of Health Stroke Scale (NIHSS) scores (range: 0 to 42, with higher scores indicating a greater deficit), diabetes, and treatment delays have been suggested to increase the risk of SICH after thrombectomy in patients with acute ischaemic stroke (AIS). [3] Poor imaging evaluations, such as low Alberta Stroke Program Early Computed Tomography Score (ASPECTS) values, slight mismatch between the infarct core and ischaemic penumbra, very low cerebral blood flow and a low Clot Burden Score (CBS), have all been reported to be associated with an increased risk of SICH. [13-16] Poor collateral circulation as assessed by the American Society of Interventional and Therapeutic Neuroradiology/Society of Interventional Radiology (ASITN/SIR) collateral score has also been found to be associated with an increased risk of $\mathrm{SICH} .[3,17]$ Additionally, reperfusion injury and device-related vessel injury are potential risk factors. [18] The inflammatory response is involved in cerebral ischaemia-reperfusion injury and plays a key role in this process, as it can direct endothelial cell injury and destroy the blood-brain barrier, resulting in cerebral haemorrhage. [19]

As illustrated by the above studies, an accurate and easily available model for predicting the occurrence of $\mathrm{SICH}$ after direct thrombectomy is still lacking. Therefore, this study aims to identify periprocedural independent risk factors and establish a novel nomogram-based model that is applicable to patients with anterior large vessel occlusion (LVO) to predict the occurrence of SICH after direct thrombectomy or bridge therapy.

\section{Methods}

\section{Patients}

The study conforms to current guidelines on the endovascular management of acute ischaemic stroke (including AHA/ASA guidelines and Chinese guidelines). All AIS patients with LVO who received endovascular procedures between November 2017 and March 2019 at the First Affiliated Hospital of Wenzhou Medical University were enrolled in the study and received treatment with intravenous tissue 
plasminogen activator (tPA) plus EVT or EVT alone. Any patient who arrived at the Emergency Department with symptom onset within 6 hours and who had received baseline CT angiography (CTA) to ensure the LVO of anterior circulation (internal carotid artery (ICA) or middle cerebral artery (MCA)) was included. Any patients with posterior circulation artery occlusions were excluded from the study. Patients were also excluded if they had a history of ICH at baseline, renal impairment (creatinine clearance level < $60 \mathrm{~mL} / \mathrm{min}$ ), contrast allergy, hypoglycaemia (serum glucose level $<2 \mathrm{mmol} / \mathrm{L}$ ), or pretreatment SBP $\geq$ $200 \mathrm{mmHg}$.

\section{Clinical data and imaging evaluation}

The following data were collected during hospitalization: age, sex, medical history of smoking and drinking, atrial fibrillation, hypertension, diabetes mellitus, NIHSS at initial presentation and dyslipidaemia. Median values were used to describe the following parameters: Pre-SBP, ASPECTS, NIHSS, CBS, ASITN/SIR, LDL, serum glucose, OTP, PTR, and OTR. In addition, mean values were used to describe the platelet count and INR. Before treatment, each patient was examined with noncontrast CT to exclude intracranial haemorrhage and to estimate ASPECTS values signing of early ischaemia. Baseline CTA was used to identify acute occlusions in the anterior circulation and the following clot characteristics: thrombus location and CBS. Before performing EVT, digital subtraction angiography (DSA) was used to evaluate the collateralization grade (ASITN/SIR) in the operating room. To guarantee the objectivity of the data, every score was graded independently by two deputy chief physicians who have extensive experience in endovascular treatment (more than 20 years, evaluating 500+ patients annually).

\section{Treatment process}

The EVT of all enrolled patients was carried out according to the new guidelines for AIS. [20] [21] Thrombectomy was performed with a stent-like retriever (Solitaire FR, Covidien, Irvine, CA) in all patients. Successful recanalization was defined as a modified Thrombolysis in Cerebral Infarction (MTICl) score of $2 \mathrm{~b}$ or 3 .

\section{Diagnosis of SICH}

Follow-up at 24-72 hours was performed with MR imaging or CT to assess whether ICH had occurred. $\mathrm{SICH}$ was diagnosed and classified according to the Heidelberg Bleeding Classification [1] by two neuroradiologists blinded to clinical information and angiographic findings. Final decisions were made by consensus.

\section{Statistical analysis}

The entire cohort was used to develop the prediction model by the statistical software R Project for Statistical Computing and SPSS 22.0 (IBM, Armonk, NY). Differences between the cohort with SICH and the cohort without SICH were assessed using the Mann-Whitney U-test for continuous variables. Categorical variables were analysed with the $\chi 2$ test or Fisher's exact test. 
To generate the nomogram, the pre-verified predictors with at least marginal significance $(P<0.05)$ in the multivariate analysis were entered into a logistic regression model. The collinearity of the combinations of variables in the training set was assessed. Regression coefficients with standard errors and odds ratios (OR) with two-sided $95 \%$ confidence intervals $(\mathrm{Cl})$ for each of the variables included in the model were calculated.

The discrimination of the nomogram was assessed by calculating the area under the receiver operating characteristic curve (AUC-ROC). Calibration of the risk prediction model was performed with the test cohort by constructing a plot comparing the observed probability of an unfavourable outcome according to the total score of the nomogram against the predicted probability based on the nomogram.

\section{Results}

Among 208 patients with EVT, 127 patients with complete data for generating the nomogram were included in the study. All 127 patients were treated with stent-like retrievers by using Solitaire FR (Covidien, Irvine, CA). The mean age of the patients was $65.9 \pm 13.1$ years, and the median baseline NIHSS score was 17.2. Of the 127 enrolled patients with AIS, 72 (56.7\%) arrived at the Emergency Department within 4.5 hours, and 61 (48\%) were eligible for intravenous thrombolysis; however, only 48 (37.8\%) received intravenous thrombolysis. ICH was diagnosed in 61 patients (48\%) within 72 hours after EVT. According to the Heidelberg Bleeding Classification, 37 patients (29.1\%) were classified as having SICH.

Univariate analysis showed that compared to patients without $\mathrm{SICH}$, in patients with $\mathrm{SICH}$, the NIHSS at initial presentation was higher (median, 22.38 versus 15.03; $\mathrm{P}<0.001$ ); the pretreatment ASPECTS was lower (median, 5.89 versus 7.69; $P<0.001$ ); the CBS was lower (median, 4.92 versus $6.01 ; P=0.005$ ); and the ASITN/SIR was lower (median, 1.62 versus $2.69 ; \mathrm{P}<0.001$ ). In addition, more patients with $\mathrm{SICH}$ had $>3$ attempted passes with retrievers during thrombectomy than patients without $\mathrm{SICH}$ (32.4\% versus 7.8\%; $\mathrm{P}<0.001)$. The clinical characteristics of all the patients are presented in Table 1. 
Table 1

Clinical characteristics of study populations

\begin{tabular}{|c|c|c|c|c|}
\hline \multirow[t]{2}{*}{ Characteristics } & \multirow[t]{2}{*}{ Total } & \multicolumn{2}{|l|}{$\mathrm{SICH}$} & \multirow[t]{2}{*}{$P$ Value } \\
\hline & & with $(n=37)$ & without(n=90) & \\
\hline Age, mean (SD), y & $65.9(13.1)$ & $67.2(9.9)$ & $65.4(14.2)$ & 0.795 \\
\hline Male sex, n (\%) & $88(69.3)$ & $27(73)$ & $61(67.8)$ & 0.564 \\
\hline Location, MCA, n (\%) & $89(70.1)$ & $26(70.3)$ & $63(70)$ & 0.976 \\
\hline Hypertension, n (\%) & $70(55.1)$ & $21(56.8)$ & $49(54.4)$ & 0.812 \\
\hline DM, n (\%) & 23(18.1) & $6(16.2)$ & 17(18.9) & 0.722 \\
\hline AF, n (\%) & 49(38.6) & 13(35.1) & $36(40.0)$ & 0.609 \\
\hline Smoking, n (\%) & 43(33.9) & 12(32.4) & $31(34.4)$ & 0.828 \\
\hline Alcohol, n (\%) & $39(30.7)$ & $10(27)$ & $29(32.2)$ & 0.564 \\
\hline Pre-anticoagulation, n (\%) & 20(15.7) & $7(18.9)$ & $13(14.4)$ & 0.529 \\
\hline \multicolumn{5}{|l|}{ Baseline measurements } \\
\hline Pre-SBP, median (IQR), mm Hg & $\begin{array}{l}150.6(128- \\
175)\end{array}$ & $\begin{array}{l}151.3(124- \\
188)\end{array}$ & 150.3(129.5-170) & 0.893 \\
\hline ASPECTS, median (IQR) & 7.17(1.78) & $5.89(1.90)$ & $7.69(1.43)$ & $<0.001^{*}$ \\
\hline NIHSS score, median (IQR) & $17.2(11-21)$ & $\begin{array}{l}22.38(14.5- \\
33.5)\end{array}$ & $15.03(10-17.3)$ & $<0.001^{*}$ \\
\hline CBS, median (SD) & $5.698(2.01)$ & $4.92(1.83)$ & $6.01(2.03)$ & $0.005^{\star}$ \\
\hline ASITN/SIR, median (SD) & $2.38(1.05)$ & $1.62(1.09)$ & $2.69(0.856)$ & $<0.001^{*}$ \\
\hline Platelet, mean (IQR), 10^9/L & $\begin{array}{l}\text { 195.0(153- } \\
231)\end{array}$ & $\begin{array}{l}181.9(142- \\
208.5)\end{array}$ & $\begin{array}{l}200.4(160.5- \\
237.1)\end{array}$ & 0.130 \\
\hline INR, mean (SD) & $1.1(0.1)$ & $1.1(0.1)$ & $1.1(0.2)$ & 0.738 \\
\hline LDL, median (IQR), mmol/L & $2.4(0.9)$ & $2.5(0.8)$ & $2.3(0.9)$ & 0.215 \\
\hline $\begin{array}{l}\text { Serum glucose, median (IQR), } \\
\mathrm{mmol} / \mathrm{L}\end{array}$ & $7.9(6-9.1)$ & $8.5(6.4-10.1)$ & 7.6(5.9-8.4) & 0.075 \\
\hline \multicolumn{5}{|l|}{ Procedure process } \\
\hline Intravenous thrombolysis, n (\%) & 48(37.8) & 18(48.6) & $30(33.3)$ & 0.106 \\
\hline OTP, median (IQR), min & $\begin{array}{l}304.6(209- \\
376)\end{array}$ & $\begin{array}{l}286.1(204- \\
367)\end{array}$ & $312.1(217-380)$ & 0.426 \\
\hline PTR, median (IQR), min & $86.7(56-112)$ & $84.2(54.5-101)$ & $87.7(54-113.3)$ & 0.979 \\
\hline
\end{tabular}




\begin{tabular}{|c|c|c|c|c|}
\hline \multirow[t]{2}{*}{ Characteristics } & \multirow[t]{2}{*}{ Total } & \multicolumn{2}{|l|}{$\mathrm{SICH}$} & \multirow[t]{2}{*}{ P Value } \\
\hline & & with $(n=37)$ & without(n=90) & \\
\hline OTR, median (IQR), min & $\begin{array}{l}390.5(303- \\
464)\end{array}$ & $\begin{array}{l}370.4(286- \\
455.5)\end{array}$ & $398.7(313.8-467)$ & 0.324 \\
\hline Passes of retriever $>3, \mathrm{n}(\%)$ & 19(15.0) & $12(32.4)$ & $7(7.8)$ & $<0.001^{*}$ \\
\hline $\mathrm{mTICl}, 2 \mathrm{~b}$ or $3, \mathrm{n}(\%)$ & $110(86.6)$ & $32(86.5)$ & $78(86.7)$ & 0.509 \\
\hline
\end{tabular}

According to the multivariate analysis, three scoring systems were identified as independent predictors: the baseline NIHSS score (OR: 1.1; 95\%Cl: $1.0-1.1 ; \mathrm{P}=0.024)$, the pretreatment ASPECT score (OR: 0.6; $95 \% \mathrm{Cl}: 0.5-0.8 ; \mathrm{P}<0.001$ ) and the ASITN/SIR collateral score (OR: $0.5 ; 95 \% \mathrm{Cl}: 0.3-0.9 ; \mathrm{P}=0.017$ ). However, we did not identify CBS (OR: $0.9 ; 95 \%$ Cl: $0.7-1.1 ; P=0.247$ ) or retriever passes >3 (OR: 1.3; $95 \% \mathrm{Cl}: 0.9-2.0 ; \mathrm{P}=0.147$ ) as independent predictors for SICH (Table 2).

Table 2

Multivariate Analysis of Predictors of SICH

\begin{tabular}{|lllll|}
\hline Variables & OR & P Value & \multicolumn{2}{c|}{$95 \%$ Wald Cl } \\
\cline { 4 - 5 } & & & lower & upper \\
\hline NIHSS & 1.1 & $0.024^{*}$ & 1.0 & 1.1 \\
\hline ASPECT & 0.6 & $0.001^{*}$ & 0.5 & 0.8 \\
\hline CBS & 0.9 & 0.247 & 0.7 & 1.1 \\
\hline ASITN & 0.5 & $0.017 *$ & 0.3 & 0.9 \\
\hline Passes $>3$ & 1.3 & 0.147 & 0.9 & 2.0 \\
\hline
\end{tabular}

To generate the nomogram for predicting the probability of $\mathrm{SICH}$, the three pretreatment scoring systems were entered into a logistic regression model. The nomogram was created by assigning a preliminary score (ranging from 0 to 40 points) for each of the three predictors, which were then summed to generate a total score and finally converted into a quantified individual risk of $\mathrm{SICH}$ after thrombectomy, as shown in Figure 1.

The AUC-ROC value of the NIHSS/ASPECT/ASITN (NAA) nomogram, which included the data from 127 patients, was 0.845 (95\% Cl: 0.7629-0.9275) (Figure 2A). The model was internally validated using 1000 bootstrap samples to calculate the discrimination and resulted in an accuracy of 0.845 (95\% Cl: $0.7629-$ 0.9273). Additionally, the calibration plots (Figure 2B) showed good agreement between the NAA nomogram predictions and actual observations.

\section{Discussion}


Our study developed and internally validated a new nomogram (NAA) using three scoring systems to predict the probability of the occurrence of $\mathrm{SICH}$ in stroke patients with anterior LVO receiving direct thrombectomy or bridge treatment.

The three scoring systems of nomogram that are widely accepted all over the world for assessing the periprocedural condition of AIS patients were all found to be independently associated with $\mathrm{SICH}$ after EVT in our study. NIHSS is a quick and simple tool for estimating the extent and severity of stroke. It is not only the most powerful predictor for the long-term outcome of patients with AIS but has also been associated with $\mathrm{SICH}$ in recent studies. $[2,14]$ In our study, baseline NIHSS scores were higher in patients with $\mathrm{SICH}$ than in patients without $\mathrm{SICH}$ (median, 22.38 versus 15.03; $\mathrm{P}<0.001$ ) and were strongly associated with SICH after thrombectomy (OR: 1.063; Cl: 1.008-1.112; P = 0.024).

ASPECT is a 10-point quantitative topographic CT scan score used in patients with MCA stroke. ASPECTS values $\leq 7$ predict a worse functional outcome at 3 months as well as symptomatic haemorrhage. [13] In our study, pretreatment ASPECT scores were lower in patients with SICH (median, 5.89 versus $7.69 ; \mathrm{P}<0.001)$ and were independently associated with SICH (OR: $0.621 ; 95 \% \mathrm{Cl}$ : $0.467-$ $0.826 ; \mathrm{P}=0.001$ ), which is consistent with the results of recent research. ASITN/SIR collateral scores, a classification for leptomeningeal compensation, were evaluated as grade 0 to 4 . A score less than 2 was associated with an increased risk of SICH after EVT. $[3,17]$ In our study, ASITN/SIR collateral scores were lower in patients with $\mathrm{SICH}$ (median, 1.62 versus $2.69 ; \mathrm{P}<0.001$ ) and independently associated with $\mathrm{SICH}$ (OR: $0.515 ; 95 \% \mathrm{Cl}: 0.299-0.877 ; \mathrm{P}=0.017$ ).

Although the three scoring systems have been validated to be independently associated with $\mathrm{SICH}$ alone, the use of combinations of the three scoring systems for predicting the occurrence of $\mathrm{SICH}$ after thrombectomy is lacking. To fill this gap, we propose the new NAA nomogram, which is a comprehensive tool that has demonstrated a higher predictive accuracy and discrimination than the three scoring systems alone. The AUC-ROC of the NAA nomogram was 0.845 (95\% Cl: $0.7629-0.9275)$, which is higher than that of the three scoring systems alone (Figure 2A). Moreover, the calibration plots of the NAA model also showed good agreement between the nomogram predictions and actual observations (Figure 2B).

The translocation of microvascular during suction of thrombus and pulling of stents, and the injury of the stents to the wall of vessels may lead to $\mathrm{ICH}$. In our study, the rate of $\mathrm{SICH}$ was higher than that reported in non-RCTs (29.1\% versus $16 \%$ ). However, SAH, a marker of procedural related haemorrhage, accounted for only $13.5 \%$ among SICH patients in our study. Ischemia-reperfusion injury and destruction of the blood-brain barrier are still considered to be the main causes of hemorrhage transformation after recanalization the blocked artery. In our study, the SICH group and the non-SICH group reaching $2 b / 3$ level are $86.5 \%$ and $86.7 \%$, respectively, for which, there is no statistical significance $(p=0.509)$. However, when the risk of $\mathrm{SICH}$ was assessed for patients with different $\mathrm{mTICl}$ scores, patients with $\mathrm{mTICl}$ score 3 had the lowest risk. $\mathrm{mTICl}$ score is an important tool to evaluate the degree of reperfusion of the blocked artery, rather than the degree of ischemia-reperfusion injury. Therefore, the $\mathrm{mTICI}$ score only reflects the association between ICH and reperfusion. Hao Y [13]reported that patients with $\mathrm{mTICI} 1$ had the highest 
risk with $\mathrm{SICH}(\mathrm{OR}, 3.62 ; 95 \% \mathrm{Cl}, 1.45-9.01$ versus patients with $\mathrm{mTICl} 3)$, followed by patients with $\mathrm{mTICl}$ 2a (OR, 2.19; 95\% Cl, 1.04-4.64) and $\mathrm{mTICl} 0(\mathrm{OR}, 1.69 ; 95 \% \mathrm{Cl}, 0.66-4.37$, overall $\mathrm{P}=0.029)$. Multivariate analysis did not identify $\mathrm{mTICI} \leq 2 \mathrm{a}$ as an independent predictor for $\mathrm{SICH}$ in his study. Dong Yang[22]conducted statistical analysis of the symptomatic intracranial hemorrhage among three groups ( $\mathrm{mTICl} 0-2 \mathrm{a}, \mathrm{mTICl} 2 \mathrm{~b}$ and $\mathrm{mTICl} 3$ ) and found there were no significant statistical significance $(\mathrm{P}=0.28)$. In fact, many other factors have an impact on the process of ischemic-reperfusion injury to influence the occurrence of $\mathrm{ICH}$, including metabolic milieu, degree of ischemic preconditioning, systemic blood pressure, blood glucose among individuals and etc.

Our study has some limitations, which should be noted. Firstly, it was based on a retrospective analysis; we plan to obtain prospective data for the next study. Secondly, although the calibration and discrimination of NAA was good, external validation in a completely different cohort is warranted. Thirdly, the sample in this study consisted of only 127 patients, which affects the reliability of these results. Finally, this study was conducted in a Chinese tertiary academic hospital. Our surgical expertise may not have been sufficient during the early phases of the study, which may have influenced the thrombectomy process. However, this situation has greatly improved as our hospital was rated as a National Emerging Comprehensive Stroke Center last year. And it is well known that Asian and Chinese populations have higher rates of sICH than other populations. What' more, due to financial reasons, most patients refused thrombolysis and more inclined to undergo thrombectomy only. Therefore, the generalizability of our results may be limited and may not be representative of all patients.

\section{Conclusions}

We have developed and internally validated a new and reliable graphical calculation instrument (NAA) that may accurately predict the possibility of SICH after thrombectomy in patients with anterior LVO. However, large-sample external validation is warranted in the future.

\section{Abbreviations}

$\mathrm{SICH}$

symptomatic intracranial haemorrhage

LVO

large vessel occlusion

EVT

endovascular treatment

NAA

NIHSS/ASPECT/ASITN

AUC

area under the curve

mRS

modified Rankin Scale 
ECASS-II

European Co-operative Acute Stroke Study-II

NINDS

National Institute of Neurological Disorders and Stroke

SITS-ISTR

Stroke-International Stroke Thrombolysis Registry

RCTs

randomized controlled trials

ASPECTS

low Alberta Stroke Program Early Computed Tomography Score

CBS

Clot Burden Score

tPA

tissue plasminogen activator

CTA

CT angiography

ICA

internal carotid artery

MCA

middle cerebral artery

DSA

digital subtraction angiography

$\mathrm{mTICl}$

modified Thrombolysis in Cerebral Infarction

OR

errors and odds ratios

$\mathrm{Cl}$

confidence intervals

\section{Declarations}

\section{Acknowledgements}

We would like to thank all the patients who participated in this study.

\section{Author Contributions}

Yunhui Wang and Qi Tu conceived the study and drafted the article. Tingting Zhao contributed to the data acquisition. Qiang Ye contributed to the diagnosis. Kuang Zheng contributed to the data analysis. 
Weizhong Zhang contributed to a major role in the interpretation of the data. Zequn Li contributed to critical revision of the article.

\section{Availability of data and materials}

The relevant anonymized patient level data are available upon reasonable request from the corresponding author, Zequn Li.

\section{Funding Sources}

This study was supported by the Natural Science Foundation of Zhejiang Province of China (LY16H090015) .

\section{Ethics approval and consent to participate}

This study was approved by the Instituitional Research Ethics Committee of First Affiliated Hospital of Wenzhou Medical University (subject number: 2019-037). All procedures performed in the studies involving human participants were in accordance with the ethical standards of our Institutional Review Board with the 1964 Helsinki Declaration and its later amendments or comparable ethical standards. In this retrospective study, the requirement for informed consent was waived by the Institutional research ethics committee of Affiliated Hospital of Wenzhou Medical University. Patient data was de-identified data upon data collection.

\section{Consent for publication}

Not applicable.

\section{Competing interests}

The authors declare that they have no conflict of interest.

\section{Author details}

${ }^{1}$ Department of Neurosurgery, The People's hospital of Deqing, Huzhou, Zhejiang Province, P.R. China 
${ }^{2}$ Department of Neurology, the First Affiliated Hospital of Wenzhou Medical

University, Wenzhou, Zhejiang Province, P.R. China

a These authors contributed equally to this study.

\section{References}

1. von Kummer R, Broderick JP, Campbell BC, Demchuk A, Goyal M, Hill MD, et al. The Heidelberg Bleeding Classification: Classification of Bleeding Events After Ischemic Stroke and Reperfusion Therapy. Stroke. 2015;46 10:2981-6; doi: 10.1161/STROKEAHA.115.010049.

2. Nogueira RG, Gupta R, Jovin TG, Levy El, Liebeskind DS, Zaidat OO, et al. Predictors and clinical relevance of hemorrhagic transformation after endovascular therapy for anterior circulation large vessel occlusion strokes: a multicenter retrospective analysis of 1122 patients. Journal of Neurolnterventional Surgery. 2015;7 1:16-21; doi: 10.1136/neurintsurg-2013-010743.

3. Hao Y, Yang D, Wang H, Zi W, Zhang M, Geng Y, et al. Predictors for Symptomatic Intracranial Hemorrhage After Endovascular Treatment of Acute Ischemic Stroke. Stroke. 2017;48 5:1203-9; doi: 10.1161/strokeaha.116.016368.

4. Berkhemer OA, Fransen PS, Beumer D, van den Berg LA, Lingsma HF, Yoo AJ, et al. A randomized trial of intraarterial treatment for acute ischemic stroke. The New England journal of medicine. 2015;372 1:11-20; doi: 10.1056/NEJMoa1411587.

5. Goyal M, Demchuk AM, Menon BK, Eesa M, Rempel JL, Thornton J, et al. Randomized assessment of rapid endovascular treatment of ischemic stroke. The New England journal of medicine. 2015;372 11:1019-30; doi: 10.1056/NEJMoa1414905.

6. Jovin TG, Chamorro A, Cobo E, de Miquel MA, Molina CA, Rovira A, et al. Thrombectomy within 8 hours after symptom onset in ischemic stroke. The New England journal of medicine. 2015;372 24:2296-306; doi: 10.1056/NEJMoa1503780.

7. Bracard S, Ducrocq X, Mas JL, Soudant M, Oppenheim C, Moulin T, et al. Mechanical thrombectomy after intravenous alteplase versus alteplase alone after stroke (THRACE): a randomised controlled trial. The Lancet Neurology. 2016;15 11:1138-47; doi: 10.1016/s1474-4422(16)30177-6.

8. Mocco J, Zaidat OO, von Kummer R, Yoo AJ, Gupta R, Lopes D, et al. Aspiration Thrombectomy After Intravenous Alteplase Versus Intravenous Alteplase Alone. Stroke. 2016;47 9:2331-8; doi: 10.1161/strokeaha.116.013372.

9. Minnerup J, Wersching H, Teuber A, Wellmann J, Eyding J, Weber R, et al. Outcome After Thrombectomy and Intravenous Thrombolysis in Patients With Acute Ischemic Stroke. Stroke. 2016;47 6:1584-92; doi: 10.1161/strokeaha.116.012619.

10. Nikoubashman O, Jungbluth M, Schürmann K, Müller M, Falkenburger B, Tauber SC, et al. Neurothrombectomy in acute ischaemic stroke: a prospective single-centre study and comparison 
with randomized controlled trials. European Journal of Neurology. 2016;23 4:807-16; doi: 10.1111/ene.12944.

11. Urra X, Abilleira S, Dorado L, Ribó M, Cardona P, Millán M, et al. Mechanical Thrombectomy in and Outside the REVASCAT Trial. Stroke. 2015;46 12:3437-42; doi: 10.1161/strokeaha.115.011050.

12. Neuberger U, Mohlenbruch MA, Herweh C, Ulfert C, Bendszus M, Pfaff J. Classification of Bleeding Events: Comparison of ECASS III (European Cooperative Acute Stroke Study) and the New Heidelberg Bleeding Classification. Stroke. 2017;48 7:1983-5; doi: 10.1161/STROKEAHA.117.016735.

13. Soize S, Barbe C, Kadziolka K, Estrade L, Serre I, Pierot L. Predictive factors of outcome and hemorrhage after acute ischemic stroke treated by mechanical thrombectomy with a stent-retriever. Neuroradiology. 2013;55 8:977-87; doi: 10.1007/s00234-013-1191-4.

14. Jiang S, Fei A, Peng Y, Zhang J, Lu YR, Wang HR, et al. Predictors of Outcome and Hemorrhage in Patients Undergoing Endovascular Therapy with Solitaire Stent for Acute Ischemic Stroke. PLoS One. 2015;10 12:e0144452; doi: 10.1371/journal.pone.0144452.

15. Mishra NK, Christensen S, Wouters A, Campbell BC, Straka M, Mlynash M, et al. Reperfusion of very low cerebral blood volume lesion predicts parenchymal hematoma after endovascular therapy. Stroke. 2015;46 5:1245-9; doi: 10.1161/STROKEAHA.114.008171.

16. Yogendrakumar V, Al-Ajlan F, Najm M, Puig J, Calleja A, Sohn SI, et al. Clot Burden Score and Early Ischemia Predict Intracranial Hemorrhage following Endovascular Therapy. AJNR American journal of neuroradiology. 2019; doi: 10.3174/ajnr.A6009.

17. Liebeskind DS, Jahan R, Nogueira RG, Zaidat OO, Saver JL. Impact of Collaterals on Successful Revascularization in Solitaire FR With the Intention for Thrombectomy. Stroke. 2014;45 7:2036-40; doi: 10.1161/strokeaha.114.004781.

18. Papanagiotou P, White CJ. Endovascular Reperfusion Strategies for Acute Stroke. JACC: Cardiovascular Interventions. 2016;9 4:307-17; doi: 10.1016/j.jcin.2015.11.014.

19. Bai J, Lyden PD. Revisiting cerebral postischemic reperfusion injury: new insights in understanding reperfusion failure, hemorrhage, and edema. Int J Stroke. 2015;10 2:143-52; doi: 10.1111/ijs.12434.

20. Powers WJ, Rabinstein AA, Ackerson T, Adeoye OM, Bambakidis NC, Becker K, et al. 2018 Guidelines for the Early Management of Patients With Acute Ischemic Stroke: A Guideline for Healthcare Professionals From the American Heart Association/American Stroke Association. Stroke. 2018;49 3:e46-e110; doi: 10.1161/STR.0000000000000158.

21. TC H, S Z, M L, Y W, J W, Q D, et al. Chinese guidelines for endovascular management of acute ischemic stroke. Chinese Journal of Stroke. 2018;13 null:1673-5763.

22. Yang D, Geng Y, Zhang M, Lin M, Shi Z, Zi W, et al. Complete Recanalization May Exert the Most Important Effect on Outcomes of Endovascular Treatment in Acute Ischemic Stroke with Small Infarct Core Beyond 6 Hours. World Neurosurg. 2019;125:e544-e51; doi:

10.1016/j.wneu.2019.01.131.

\section{Figures}




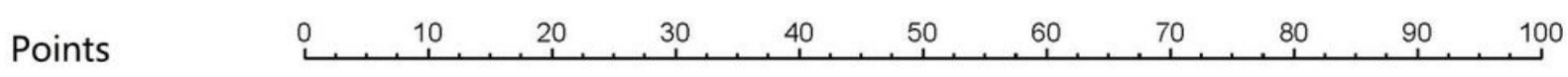

NIHSS

\begin{tabular}{|c|c|c|c|c|c|c|c|}
\hline$\Gamma$ & $T$ & $T$ & $T$ & $T$ & $T$ & $T$ & $T$ \\
\hline 0 & 5 & 10 & 15 & 20 & 25 & 30 & 35 \\
\hline
\end{tabular}

ASPECT

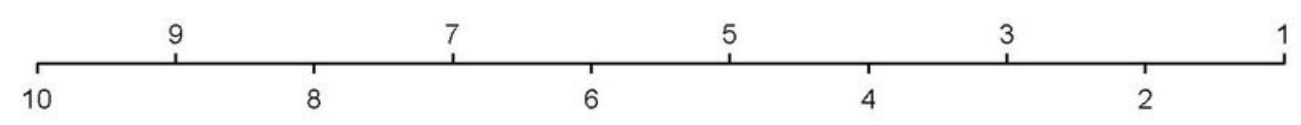

ASITN

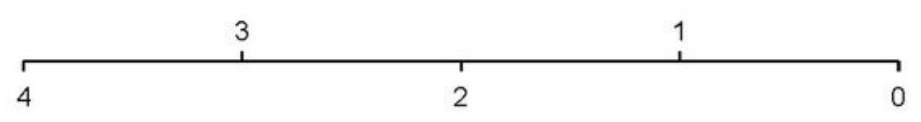

Total Points

\begin{tabular}{|c|c|c|c|c|c|c|c|c|c|c|c|}
\hline 0 & 20 & 40 & 60 & 80 & 100 & 120 & 140 & 160 & 180 & 200 & 220 \\
\hline
\end{tabular}

$\mathrm{SICH}$

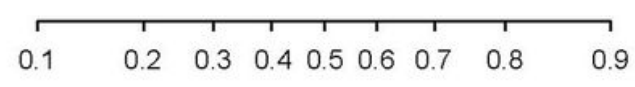

\section{Figure 1}

NAA nomogram plot. NIHSS, National Institute of Health Stroke Scale; ASPECTS, Alberta Stroke Program Early Computed Tomography Score; ASITN/SIR, American Society of Interventional and Therapeutic Neuroradiology/ Society of Interventional Radiology. 
A

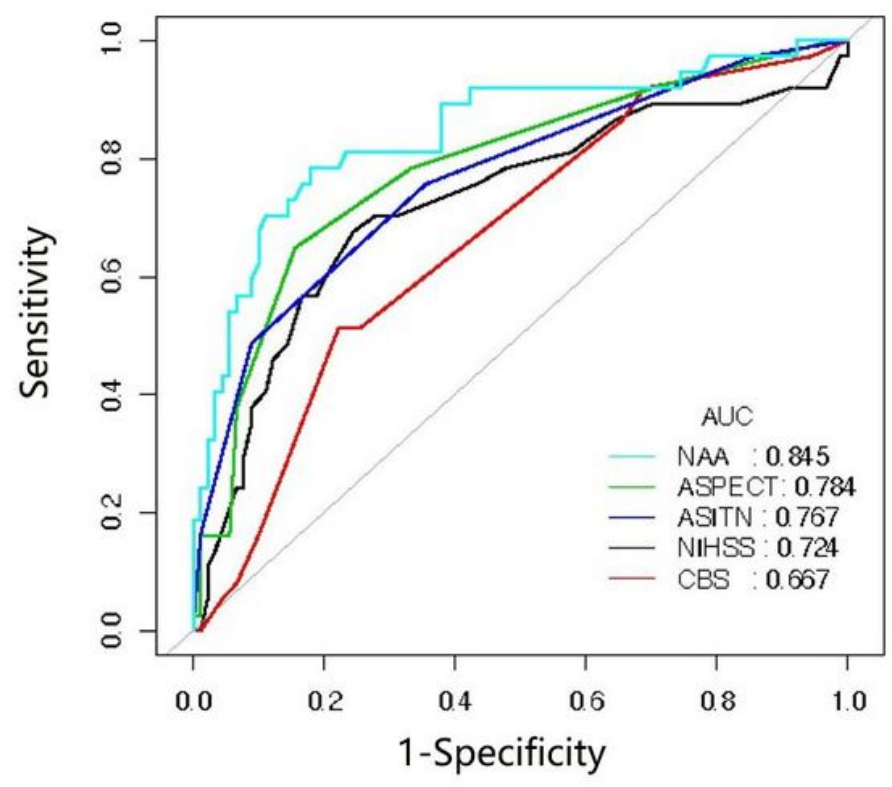

B

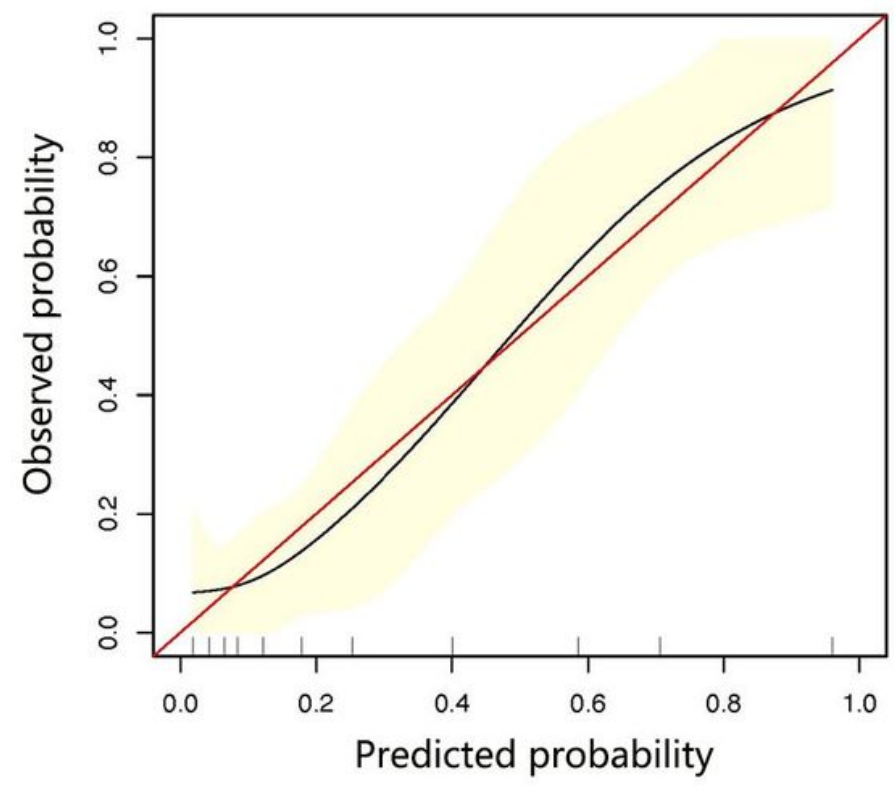

Figure 2

पADROC curve for SICH $\mathrm{BB}$ CCalibration curve. ASPECTS, Alberta Stroke Program Early Computed Tomography Score; ASITN/SIR, American Society of Interventional and Therapeutic Neuroradiology/ Society of Interventional Radiology

; NIHSS, National Institute of Health Stroke Scale; NAA: the prediction model based on NIHSS, ASPECT, and ASITN/SIR; CBS, Clot Burden Score.

\section{Supplementary Files}

This is a list of supplementary files associated with this preprint. Click to download.

- data.xlsx 